\title{
Experience of more than 100 preimplantation genetic diagnosis cycles for monogenetic diseases using whole genome amplification and linkage analysis in a single centre
}

\author{
Judy FC Chow, William SB Yeung *, Vivian CY Lee, Estella YL Lau, PC Ho, Ernest HY Ng
}

\section{A B S T R A C T}

Objective: To report the outcomes of more than 100 cycles of preimplantation genetic diagnosis for monogenetic diseases.

Design: Case series.

Setting: Tertiary assisted reproductive centre in Hong Kong, where patients needed to pay for the cost of preimplantation genetic diagnosis on top of standard in-vitro fertilisation charges.

Patients: Patients undergoing preimplantation genetic diagnosis for monogenetic diseases at the Centre of Assisted Reproduction and Embryology, Queen Mary Hospital-The University of Hong Kong between 1 August 2007 and 30 April 2014 were included.

Interventions: In-vitro fertilisation, intracytoplasmic sperm injection, embryo biopsy, and preimplantation genetic diagnosis.

Main outcome measures: Ongoing pregnancy rate and implantation rate.

Results: Overall, 124 cycles of preimplantation genetic diagnosis were initiated in 76 patients, 101 cycles proceeded to preimplantation genetic diagnosis, and 92 cycles had embryo transfer. The ongoing pregnancy rate was $28.2 \%$ per initiated cycle and $38.0 \%$ per embryo transfer, giving an preimplantation genetic diagnosis, cryopreserved embryos were replaced resulting in an ongoing pregnancy rate of $37.5 \%$ and implantation rate of $30.0 \%$. The cumulative ongoing pregnancy rate was $33.1 \%$. The most frequent indication for preimplantation genetic diagnosis was thalassaemia, followed by neurodegenerative disorder and cancer predisposition. There was no misdiagnosis.

Conclusions: Preimplantation genetic diagnosis is a reliable method to prevent couples conceiving fetuses severely affected by known genetic disorders, with ongoing pregnancy and implantation rates similar to those for in-vitro fertilisation for routine infertility treatment.

\section{Hong Kong Med J 2015;21:299-303}

\section{DOI: 10.12809/hkmj144436}

\section{JFC Chow, MPhil}

${ }^{1}$ WSB Yeung *, PhD

${ }^{2}$ VCY Lee, FHKAM (Obstetrics and Gynaecology)

${ }^{2}$ EYL Lau, PhD

${ }^{1}$ PC Ho, FRCOG, FHKAM (Obstetrics and Gynaecology)

1 EHY Ng, FRCOG, FHKAM (Obstetrics and Gynaecology)

1 Department of Obstetrics and Gynaecology, The University of Hong Kong, Queen Mary Hospital, Pokfulam, Hong Kong

2 Department of Obstetrics and Gynaecology, Queen Mary Hospital, Hong Kong

* Corresponding author: wsbyeung@hku.hk

New knowledge added by this study

Preimplantation genetic diagnosis is feasible and reliable for at least 20 genetic conditions in Hong Kong.

Implications for clinical practice or policy

Preimplantation genetic diagnosis should be considered an alternative method in preconception counselling for couples at risk for a serious genetic disease.

\section{Introduction}

Preimplantation genetic diagnosis (PGD) refers to the determination of genotype of embryos before transfer during in-vitro fertilisation (IVF) cycles. The technique can prevent couples at risk for a serious genetic disease from having an affected fetus, and therefore protects couples from the psychological trauma associated with carrying an affected child, termination of pregnancy, or recurrent miscarriage.
During PGD, one blastomere is biopsied from each day-3 embryo (6-8 cells) and, after whole genome amplification (WGA), mutations can be detected directly by minisequencing and/or indirectly by linkage analysis. The first PGD baby was born in 1989 following PGD for a sex-linked genetic disease using polymerase chain reaction (PCR) for sex determination. ${ }^{1}$

According to a recent report of the European 


\section{利用全基因組擴增和連鎖分析為單基因疾病進行 逾百個胚胎植入前遺傳學診斷週期 \\ 㱀鳳翔、楊樹標、李芷茵、劉綺蘭、何柏松、吳鴻裕 \\ 目的：報告逾百個胚胎植入前遺傳學診斷（PGD）在單基因疾病週期 的結果。}

設計：病例系列研究。

安排：香港一所設有體外受精以及PGD收費服務的大學醫院輔助生育 中心。

患者：2007年8月 1 日至2014年4月30日期間，在香港大學瑪麗醫院輔 助生育中心對單基因疾病進行PGD的患者。

介入：體外受精（IVF）、卵胞漿內單精子注射（ICSI）、胚胎活檢 和PGD。

主要結果測量 : 持續妊娠率及著床率。

結果：研究期間 76位婦女為PGD進行了 124 個週期的超排卵, 其中 101 例成功進行PGD，92例進行了胚胎移植。持續妊娠率為每超排卵 週期 $28.2 \%$, 及每移植週期 $38.0 \%$, 著床率為 $35.2 \%$ 。經PGD診斷為正 常的胚胎, 經過凍存後進行了 16 次凍融肧胎移植週期, 持續妊娠率為 $37.5 \%$, 著床率 $30.0 \%$ 。累計持續妊娠率為每超卵排週期 $33.1 \%$ 。最常 見進行PGD的單基因疾病是地中海貧血、神經退化性疾病和癌症的易 感性。至今沒有誤診。

結論：PGD是一種可靠的方法, 以避免夫婦懷有受嚴重遺傳病影響的 胎兒。經過PGD篩選後的持續妊娠率及著床率跟那些接受常規IVF治 療不孕症的週期相若。
The data of all PGD cycles for monogenetic diseases performed in the Department of Obstetrics and Gynaecology, Queen Mary Hospital/The University of Hong Kong from 1 August 2007 to 30 April 2014 were retrieved. Depending on the monogenetic disease, the definitive mutation(s) responsible for the disease were confirmed in accredited genetic laboratories, including the Department of Pathology, Queen Mary Hospital; Clinical Genetic Service, Department of Health, Hong Kong SAR; and Molecular Pathology Division, Hong Kong Sanatorium \& Hospital. Preimplantation genetic diagnosis was offered to couples with a defined genetic disease, irrespective of whether the couples had a previous affected pregnancy. All couples were extensively counselled by the reproductive medicine subspecialists and a clinical geneticist on the potential risks of IVF, intracytoplasmic sperm injection (ICSI), and PGD. The couples decided whether to proceed to PGD after non-directive informative counselling. They were also advised to have a confirmatory prenatal diagnosis for the ensuing pregnancy. Depending on the availability of the test, couples could choose an invasive or non-invasive prenatal diagnostic test to confirm the diagnosis by an accredited genetic laboratory different from the PGD laboratory.

\section{Treatment regimen}

The details of the protocols for the ovarian stimulation regimen, gamete handling, and frozenthawed embryo transfer (FET) have been previously described. ${ }^{6}$ Surplus good-quality embryos unaffected by monogenetic diseases were vitrified by the CVM Vitrification System (CryoLogic, Mulgrave, Australia). If the patient did not become pregnant in the fresh cycle, the vitrified embryos were warmed and replaced in a subsequent FET cycle.

\section{Preimplantation genetic diagnosis}

The HKU-QMH CARE has been performing PGD for about 10 years. The procedures for PGD have been previously described. ${ }^{5}$ In brief, embryo biopsy was performed on day 3 at the 6 to 8 cells stage, with one blastomere biopsied. Whole genome amplification by multiple displacement amplification was performed on a single blastomere. ${ }^{5}$ In all the PGD cases for monogenetic diseases, apart from direct mutation detection (except for those involving large deletions), linkage analysis was performed by two to 10 microsatellite markers flanking the mutation to reduce possible errors due to allelic dropout. Aneuploidy was not determined because of lack of indications. When required, human leukocyte antigen (HLA) typing was performed by PCR-based sequence specific primer (Collaborative Transplant Study, University of Heidelberg, Heidelberg, Germany) in the same setting as for PGD. 


\section{Results}

Between 1 August 2007 and 30 April 2014, 76 couples initiated 124 cycles for monogenetic diseases. The median age of the women was 35 (range, 26-41) years. Embryo biopsy and PGD were performed in 101 cycles, including three cycles of combined HLA typing and beta-thalassaemia. The mean number of embryos biopsied per oocyte retrieval cycle was 6.1 (761/124). A total of 761 blastomeres were biopsied and a conclusive diagnosis was obtained for 692 (91\%) blastomeres. An inconclusive diagnosis during PGD could be due to failure in WGA or aneuploidy. Preimplantation genetic diagnosis was cancelled in $23(18.5 \%)$ cycles after initiation of stimulation because of poor responses (13 cycles), risk for ovarian hyperstimulation syndrome (OHSS; 4 cycles), no mature oocytes available (3 cycles), failed fertilisation (2 cycles), or an embryologist was unavailable for PGD (1 cycle). In case of poor response (<4 good-quality embryos on day 3$)$, cleavage-stage embryos were frozen, subsequently thawed, and pooled with fresh embryos from the following stimulated cycle for PGD. When there was excessive ovarian response and a patient was at risk for OHSS, all cleavage-stage embryos were cryopreserved and PGD was performed in the hormone replacement treatment cycles.

Overall, 92 PGD cycles proceeded to embryo transfer with one or two blastocysts replaced (mean, 1.8), resulting in an ongoing pregnancy rate (pregnancy beyond 8-10 weeks of gestation) of $28.2 \%$ per initiated cycle, $38.0 \%$ per transfer, and implantation rate of $35.2 \%$ (Table 1). Embryo transfer was cancelled in nine $(8.9 \%)$ cycles after PGD due to no genetically transferrable embryos available (4 cycles), no HLA-matched embryo (1 cycle), or patient at risk for OHSS (4 cycles). From August 2012 onwards, all genetically transferrable embryos were cryopreserved after PGD when patients were at risk for OHSS.

There were 16 cycles of natural-cycle FET for PGD blastocysts, resulting in an ongoing pregnancy rate of $37.5 \%$ per transfer. The mean number of blastocysts replaced in the FET cycle was 1.3. The implantation rate in the FET cycle was $30.0 \%$. The cumulative ongoing pregnancy rate was $33.1 \%$ per initiated cycle (Table 1).

The indications for PGD are listed in Table 2. The most frequent indication for PGD was thalassaemia (70.2\%), followed by spinocerebellar ataxia type 3 (SCA3; 4.8\%), and HD (4.0\%). Preimplantation genetic diagnosis was performed for 20 monogenetic diseases. Successful pregnancy was achieved after PGD for 14 genetic diseases. The pregnant women were referred to the maternal-fetal medicine team at Tsan Yuk Hospital for counselling and confirmation of the genetic diseases by prenatal diagnosis or postnatal cord blood genetic tests.
TABLE I. Results of preimplantation genetic diagnosis in fresh and frozen-thawed embryo transfer cycles

\begin{tabular}{|c|c|c|}
\hline & Fresh ET & Frozen-thawed ET \\
\hline No. of initiated cycles & 124 & - \\
\hline No. of PGD performed & 101 & - \\
\hline No. of ET & 92 & 16 \\
\hline Mean No. of embryos replaced per ET & 1.8 & 1.3 \\
\hline OPR per initiated cycle & $28.2 \%$ & - \\
\hline OPR per ET & $38.0 \%$ & $37.5 \%$ \\
\hline Implantation rate & $35.2 \%$ & $30.0 \%$ \\
\hline Cumulative OPR per initiated cycle & \multicolumn{2}{|c|}{$33.1 \%$} \\
\hline
\end{tabular}

Abbreviations: $\mathrm{ET}=$ embryo transfer; OPR = ongoing pregnancy rate (pregnancy beyond 8-10 weeks of gestation); PGD = preimplantation genetic diagnosis

TABLE 2. List of preimplantation genetic diagnosis cycles for monogenetic diseases

\begin{tabular}{|c|c|c|c|}
\hline & $\begin{array}{l}\text { No. of } \\
\text { cycles }\end{array}$ & $\begin{array}{c}\text { Mode of } \\
\text { inheritance }\end{array}$ & Live birth \\
\hline \multicolumn{4}{|l|}{ Blood disorder } \\
\hline Haemophilia A & 1 & X-linked recessive & No \\
\hline Alpha-thalassaemia & 64 & Autosomal recessive & Yes \\
\hline Beta-thalassaemia \pm HLA & 23 & Autosomal recessive & Yes \\
\hline \multicolumn{4}{|l|}{ Neurodegenerative disorder } \\
\hline Huntington's disease & 5 & Autosomal dominant & Yes \\
\hline Spinocerebellar ataxia type 3 & 6 & Autosomal dominant & Yes \\
\hline \multicolumn{4}{|l|}{ Cancer predisposition } \\
\hline Breast cancer (BRCA2) & 1 & Autosomal dominant & Yes \\
\hline Familial adenomatous polyposis & 2 & Autosomal dominant & Yes \\
\hline Neurofibromatosis type I & 1 & Autosomal dominant & Yes \\
\hline Pheochromocytoma & 1 & Autosomal dominant & No \\
\hline von Hippel-Lindau disease & 1 & Autosomal dominant & No \\
\hline \multicolumn{4}{|l|}{ Muscular dystrophy } \\
\hline BMD/DMD & 3 & X-linked recessive & Yes \\
\hline Myotonic dystrophy & 2 & Autosomal dominant & No \\
\hline \multicolumn{4}{|l|}{ Connective tissue/skeletal disorder } \\
\hline Osteogenesis imperfecta & 3 & Autosomal dominant & No \\
\hline Spondyloperipheral dysplasia & 1 & Autosomal dominant & Yes \\
\hline \multicolumn{4}{|l|}{ Metabolic disorder } \\
\hline Metachromatic leukodystrophy & 1 & Autosomal recessive & Yes \\
\hline Propionic acidaemia & 1 & Autosomal recessive & Yes \\
\hline \multicolumn{4}{|l|}{ Others } \\
\hline Juvenile retinoschisis & 1 & X-linked recessive & Yes \\
\hline Opitz G/BBB syndrome & 1 & X-linked recessive & No \\
\hline Synpolydactyly & 3 & Autosomal dominant & Yes \\
\hline Tuberous sclerosis & 2 & Autosomal dominant & Yes \\
\hline Waardenburg syndrome type I & 1 & Autosomal dominant & No \\
\hline
\end{tabular}

Abbreviations: BMD = Becker muscular dystrophy; DMD = Duchenne muscular dystrophy; HLA = human leukocyte antigen 
The latter was chosen by some of the patients who worried about the risk of miscarriage associated with invasive prenatal tests. Based on the available results of the confirmation genetic tests, no misdiagnosis was found in this small series.

\section{Discussion}

In 2014, the ESHRE PGD Consortium published data for 1597 PGD cycles from 60 PGD centres in Europe in 2009. ${ }^{7}$ When comparing our PGD data with those of the ESHRE PGD Consortium, we have a comparable mean number of embryos biopsied per oocyte retrieval cycle (6.1 vs 6.6$)$ and similar mean number of embryo transfers per retrieval cycle (1.4 vs 1.3). The ESHRE PGD Consortium reported a clinical pregnancy rate of $30.2 \%$ per transfer, while the ongoing pregnancy rate per transfer in our centre was $38.0 \%$ for fresh transfer and $37.5 \%$ for FET. The ongoing pregnancy rate per transfer in the IVFICSI cycle without PGD was $36 \%$ in our centre. The implantation rate of PGD embryos from the ESHRE PGD Consortium was $21.3 \%$ and in our programme was $35.2 \%$ for fresh cycles and $30.0 \%$ for FET cycles. The implantation rate in our IVF-ICSI programme without PGD was $27.7 \%$.

A limitation of the present study was that the number of cases of each genetic disease involved was small. Many genetic diseases had only one case in this cohort of patients. The usefulness of PGD in these cases needs to be confirmed in a larger cohort of patients.

The cancellation rate for PGD after initiation of stimulation was $18.5 \%$ (23/124). The major reason for cancellation was poor response leading to a small number of good-quality embryos available for PGD. In such circumstance, cleavage-stage embryos were frozen and batched for the next stimulated PGD cycle. By increasing the number of embryos tested per PGD cycle, this 'batching' strategy increased the chance of having disease-free embryos for transfer. The strategy also enabled patients to have the bestquality embryo chosen, instead of experiencing multiple cancellations of embryo transfer after PGD.

For those cases proceeding to PGD, 8.9\% resulted in no embryo transfer. Embryo transfer was cancelled because of the risk for OHSS or no genetically transferable embryos available. In cases at risk for OHSS, good-quality blastocysts were vitrified for subsequent FET cycles. Some reports have suggested that FET cycles may result in higher pregnancy and implantation rates than stimulated cycles $^{8-10}$ due to the better receptivity of the endometrium without gonadotropin stimulation. An additional benefit of FET lies in being free of risk for OHSS when compared with fresh embryo transfer.

Among the 124 cycles initiated for PGD during the study period, alpha- and beta-thalassaemia (autosomal recessive disorders) accounted for
$70.2 \%$ of the PGD cases. This is due to a high percentage of carriers in the population in Hong Kong, with a prevalence of $4.5 \%$ and $2.8 \%$ for alphathalassaemia (South-East Asian deletion type) and beta-thalassaemia, respectively. ${ }^{11,12}$ The second most frequent indication for PGD was neurodegenerative disorders such as SCA3 and HD, which are inherited in an autosomal dominant fashion. These conditions accounted for $8.9 \%$ of PGD cycles. The local prevalence of HD is estimated to be four per million population. ${ }^{13,14}$ There were 16 SCA cases diagnosed in three hospitals in Hong Kong within 3 years, and SCA3 accounted for $75 \%$ of the cases. ${ }^{15}$

It is noteworthy that $4.8 \%$ of the PGD cases were for cancer predisposition. However, it is always controversial to offer PGD to couples with inherited mutations of late-onset reduced penetrance cancer predisposition such as breast cancer. ${ }^{16}$ Additional controversy lies in the fact that PGD does not remove all the risks associated with the disease. Other known risk factors involved in breast cancer include obesity, use of hormone therapies (progestin and oestrogen), increased breast tissue density, alcohol use, and physical inactivity. ${ }^{17}$ In 2003, the ESHRE Ethics Task Force published a recommended multidisciplinary approach to the application of PGD, stating that PGD for multifactorial diseases such as $B R C A$ mutation is acceptable notwithstanding the uncertainties about the genetic predisposition and the epigenetic influence. ${ }^{18}$ The United Kingdom Human Fertilisation and Embryology Authority also accepted PGD for various hereditary cancer syndromes, including familial adenomatous polyposis, neurofibromatosis type 1 and type 2 , and von Hippel-Lindau syndrome.

Apart from the monogenetic diseases, other genetic tests such as HLA typing can be done on the same samples. In these cases, the embryos were not treated differently from those without HLA typing. Therefore, it is unlikely that the additional test would affect the ongoing pregnancy rate or implantation rate. Without indication, aneuploidy screening was not performed at the same time as PGD for monogenetic diseases. In our series, there were two pregnancies complicated by aneuploidy (trisomy 21 and trisomy 13 for each). Both patients were young (age, 31 years) with no previous pregnancy affected by aneuploidy. The usefulness of preimplantation aneuploidy screening in young patients is still under debate.

In our centre, we always consider each case individually and take into account the family histories of the couples, especially for late-onset genetic diseases such as SCA3 and HD and those with reduced penetrance and multifactorial cancer predisposition such as breast cancer. Patients were extensively counselled by a geneticist before referral for PGD treatment. Informative and non-directive 
counselling by specialists in reproductive medicine was also given to patients. Options other than PGD-such as prenatal diagnosis after becoming pregnant without PGD, gamete donation, embryo donation, adoption, or remaining childless-were discussed. Patients who became pregnant after PGD were referred to the prenatal diagnosis centre in Tsan Yuk Hospital for counselling. Prenatal diagnosis was encouraged for confirmation of the genetic status of the fetus. If patients refused to undergo invasive prenatal testing, postnatal cord blood genetic confirmation may be considered if the offspring will benefit from the early surveillance or postnatal treatment. When encountering controversial PGD cases, meetings were held to discuss the case with geneticists, obstetricians, specialists in prenatal diagnosis, ethicists, and other relevant specialists such as oncologists before offering PGD.

\section{Conclusions}

Preimplantation genetic diagnosis is a reliable method with ongoing pregnancy rate and implantation rate similar to those with IVF and ICSI. When couples have known genetic diseases, they should be counselled before pregnancy for preimplantation genetic diseases as an alternative to prenatal diagnosis, even when they do not have a previous affected pregnancy.

\section{Acknowledgements}

We would like to thank the patients, nurses, clinicians, technicians, and embryologists at the Centre of Assisted Reproduction and Embryology, Queen Mary Hospital-The University of Hong Kong for their contribution in the PGD programme.

\section{Declaration}

The authors declare that they have no conflict of interest.

\section{References}

1. Handyside $\mathrm{AH}$, Kontogianni EH, Hardy K, Winston RM. Pregnancies from biopsied human preimplantation embryos sexed by Y-specific DNA amplification. Nature 1990;344:768-70.

2. Harper JC, Wilton L, Traeger-Synodinos J, et al. The ESHRE PGD Consortium: 10 years of data collection. Hum Reprod Update 2012;18:234-47.

3. Chan V, Ng EH, Yam I, Yeung WS, Ho PC, Chan TK. Experience in preimplantation genetic diagnosis for exclusion of homozygous alpha degrees thalassemia.
Prenat Diagn 2006;26:1029-36.

4. Hellani A, Coskun S, Benkhalifa $M$, et al. Multiple displacement amplification on single cell and possible PGD applications. Mol Hum Reprod 2004;10:847-52.

5. Chow JF, Yeung WS, Lau EY, et al. Singleton birth after preimplantation genetic diagnosis for Huntington disease using whole genome amplification. Fertil Steril 2009;92:828. e7-10.

6. Ng EH, Yeung WS, Lau EY, So WW, Ho PC. High serum oestradiol concentrations in fresh IVF cycles do not impair implantation and pregnancy rates in subsequent frozenthawed embryo transfer cycles. Hum Reprod 2000;15:2505.

7. Moutou C, Goossens V, Coonen E, et al. ESHRE PGD Consortium data collection XII: cycles from January to December 2009 with pregnancy follow-up to October 2010. Hum Reprod 2014;29:880-903.

8. Evans J, Hannan NJ, Edgell TA, et al. Fresh versus frozen embryo transfer: backing clinical decisions with scientific and clinical evidence. Hum Reprod Update 2014;20:80821.

9. Roque M, Lattes K, Serra S, et al. Fresh embryo transfer versus frozen embryo transfer in in vitro fertilization cycles: a systematic review and meta-analysis. Fertil Steril 2013;99:156-62.

10. Shapiro BS, Daneshmand ST, Garner FC, Aguirre M, Hudson C. Clinical rationale for cryopreservation of entire embryo cohorts in lieu of fresh transfer. Fertil Steril 2014;102:3-9.

11. Lau YL, Chan LC, Chan YY, et al. Prevalence and genotypes of alpha- and beta-thalassemia carriers in Hong Kongimplications for population screening. $\mathrm{N}$ Engl J Med 1997;336:1298-301.

12. Sin SY, Ghosh A, Tang LC, Chan V. Ten years' experience of antenatal mean corpuscular volume screening and prenatal diagnosis for thalassaemias in Hong Kong. J Obstet Gynaecol Res 2000;26:203-8.

13. Leung CM, Chan YW, Chang CM, Yu YL, Chen CN. Huntington's disease in Chinese: a hypothesis of its origin. J Neurol Neurosurg Psychiatry 1992;55:681-4.

14. Chang CM, Yu YL, Fong KY, et al. Huntington's disease in Hong Kong Chinese: epidemiology and clinical picture. Clin Exp Neurol 1994;31:43-51.

15. Lau KK, Lam K, Shiu KL, et al. Clinical features of hereditary spinocerebellar ataxia diagnosed by molecular genetic analysis. Hong Kong Med J 2004;10:255-9.

16. Konstantopoulou I, Pertesi M, Fostira F, Grivas A, Yannoukakos D. Hereditary cancer predisposition syndromes and preimplantation genetic diagnosis: where are we now? J BUON 2009;14 Suppl 1:S187-92.

17. Emens LA, Jaffee EM. Leveraging the activity of tumor vaccines with cytotoxic chemotherapy. Cancer Res 2005;65:8059-64.

18. Shenfield F, Pennings G, Devroey P, Sureau C, Tarlatzis B, Cohen J; ESHRE Ethics Task Force. Taskforce 5: preimplantation genetic diagnosis. Hum Reprod 2003;18:649-51. 